\title{
Processes for managing project uncertainty with contingency planning
}

\author{
Cathy Roberts, Chris Wild, Kurt Maly \\ Old Dominion University Department of Computer Science \\ Norfolk, VA, USA, Phone: (804) 683-3915, FAX: (804) 683-4900, \\ [ccr|wild|maly]@cs.odu.edu
}

\begin{abstract}
One of the most pervasive problems faced by project managers is controlling a large project in an uncertain and distributed work environment. We believe that many of the inherent difficulties in managing large engineering and software projects result from difficulties in managing the uncertainty inherent in any lengthy, complex undertaking. Currently available project management tools allow the project manager to perform "what-if" and risk analyses, but do not support the management of an uncertain project environment. Existing decision support systems allow analysis of known facts about the project, but they focus upon what should be done with information after it is available. They do not support decision-making in the face of evolving and often insufficient project information, and they do not support the decision-maker when a plan must be enacted even before a decision can be made. Also, they assume that decisions are made once, instantaneously, and in isolation from the external project environment.

In our approach, we respond to the presence of project uncertainty by supporting the manager in developing and enacting competitive contingency plans. Competitive contingency plans are those plans which solve a particular project objective, and which are performed concurrently because sufficient information is not available to allow the manager to choose the single most appropriate plan. The competing plans are simultaneously enacted because project uncertainty precludes selection of a single correct course of action, and the manager must proceed with project tasks based only on the information at hand. In this paper, we address the management of contingency plans in the inherently uncertain environment of large-scale projects. Our major objectives for this research are to define and implement a process for decision-based contingency planning, and a knowledge structure to support this process. By utilizing this decision process model, we will be able to see the effects of new information upon the project and communicate the new information to affected project team members.
\end{abstract}




\section{Keywords}

Project management, decision-based system development, contingency planning, group communication, uncertainty

\section{INTRODUCTION}

Organizations are becoming increasingly linked to their information systems, and this "cyberorganization" provides a diverse set of services which are distributed in space and time. These services include engineering services (such as those provided by consultants), consumer services (such as those provided by stock brokers), consumer products (services provided by manufacturers), health care services, and so on. Management of system development projects in these various domains is impeded by many pervasive problems, and many of them involve uncertainty about the project and its environment. Projects have evolving goals, and have constraints introduced by agents external to the project. Because the cyberorganization's services are becoming more distributed and complex, the systems supporting the cyberorganization are also becoming larger and more diverse. They are developed by team members who may be distributed across wide geographic areas, and who often utilize a distributed information architecture. Also, this team is often composed of consultants or contract workers whose services are outsourced. The system development process must support complex collaborative efforts for a complex user, the cyberorganization. It also must support the uncertainty which results from this distributed, complex nature of the cyberorganization. Each step of this process involves decision management by team members and managers, regardless of their roles in the project or their geographic location. The impact of decisions upon each other needs to be represented, and changes which impact a decision need to be effectively communicated. Management of such a development process in the face of uncertainty requires the ability to understand and review the evolving web of decisions which results in the product which is being generated.

Support for project management has previously borrowed from techniques for managing traditional engineering projects. The structure of the organization which guides the project decision management process is often pictured as hierarchical or matrix-based. However, structuring the organization around the project decision-making process is a more appropriate approach when the projects involve a significant amount of problem-solving. The decision process guides the development of products for the cyberorganization, and this process should be reflected in the support for the organizational structure. The stakeholders in any single decision are a diverse group which often cut across organization boundaries (and may indeed involve members outside of the organization).

Traditional project management techniques also fall short in an attempt to manage projects for the cyberorganization. Techniques which allow the management of project budgets are available (traditional bookkeeping and accounting techniques, for example), as are good scheduling techniques and resource management methods. Decision support systems are effective in analyzing decisions when relevant project information has been gathered and is readily available. However, none of these methods provides integrated support for the ongoing decision processes which drive the management of the uncertain project environment.

To deal with uncertainty, a manager often must be creative with his or her approach to solving problems and to project tasking, and will likely fall back on techniques such as 
contingency planning if he or she does not have sufficient information to select a single course of action from among many alternatives. None of the methods mentioned in the preceding paragraph was designed to continuously handle project uncertainties. The research presented in this paper identifies a process for managing competitive contingency plans when evolving project requirements exist. We do not propose a decision-theoretic approach, since we could not adequately deal with uncertainty in this manner.

Our approach for incorporating decision process support into project management has its roots in the Decision-based System Development (DBSD) paradigm. DBSD was developed at Old Dominion University to manage and record system development decisions and the artifacts which result from the development process (Wild and Maly, 1992). Its focus is upon the process of decision-making, as opposed to simply building the decision tree. Its initial application was to software projects which are small in scope (contract value of less than $\$ 100,000)$; however, it has also been shown to be useful in managing more complex problems (Wild et. al., 1994). DBSD views system development as a series of problem-solving episodes which determines the characteristics of the system which is ultimately created. There are a large number of these episodes in any cyberorganization which much be managed, and they are partly driven by their distributed nature, the uncertainty of available information, the complexity of the project, and a need to take action before the scope of the project can be fully determined. Since decisions are not made once, forever, and instantaneously, it is necessary to provide support for managing decisions over time. DBSD emphasizes the need to support the key process elements (decisions) which lead to the design of the final product structure.

DBSD has been applied to five different system development projects. These applications span all phases of the software life-cycle. Most recently, the paradigm has been applied to NASA's Low-Visibility Landing and Surface Operations (LVLASO), and to the day-to-day management of the development of software tools supporting the Advanced Research Project Agency's (ARPA's) large distributed simulation software product called ModSAF. The LVLASO application focused on capturing the development process in its earliest phases by recording the decisions which defined the direction of the project. The application to ModSAF tracked the daily management decisions which guide the development of new ModSAF-related software tools. The focus of this application is upon project management.

\section{A CONCEPTUAL MODEL FOR MANAGING UNCERTAINTY WITH CONTINGENCY PLANS}

Decision-making plays roles in many facets of the management of system development projects. These decisions also have several characteristics common among these facets. They are not made instantaneously, since it often takes considerable time to identify alternatives, assign criteria for evaluating them, and then make the evaluation. For example, it may be necessary to build a simulation in order to better understand the nature of the problem being solved. The decisions are also not made in isolation. External factors often affect the choice which must be made from among alternatives. These factors include previous decisions made on the project which define the context in which subsequent decisions take place as well as environmental factors (such as changing government regulations, or new products from competitors or suppliers). Also, decisions are not always made exactly once. They are 
regularly revisited during a project, and are also relevant to other projects and can be reused. Because of these three characteristics of project decisions, uncertainty is prevalent in the project's scope. At any given point, information is being gathered for decision-making, decisions are being reviewed and revised, and the external project environment is changing. This uncertainty is not typically the result of bad project management practices, but does result from changing customer requirements, market conditions, and other factors outside the control of the project manager (Kraut and Streeter, 1995). This uncertainty causes the manager to utilize techniques such as conditional decision-making, risk analysis, and contingency planning. Our focus is upon managing competitive contingency plans, and is described in the following section.

\subsection{Competing Contingency Plans}

Contingency plans are often enacted when not enough information is available to make an informed decision, but tasks must begin anyway. In other words, they involve the paradoxical enactment of a decision before the decision is actually made. A plan is chosen contingent upon the receipt of some outstanding information, or perhaps upon the occurrence of some event. Although contingency planning can be supported by traditional project management methods such as PERT (Winstanley, 1991), there is a need to provide support for contingency planning which is more dynamic and decision-based than the traditional methods. A PERT view of a contingency plan would, for example, show the tasks being performed in parallel in the PERT network. PERT and CPM, however, provide a view of a project's status at a discrete point in time and do not show the decisions which resulted in the current status. They also do not allow the analyst to examine tradeoffs among contingency plans, the trigger points (i.e., events or decisions which allow the selection of one course of action from among the contingencies) which enact plans, and the risk involved in pursuing the contingencies. While contingency plans can be represented by these methods, they are not distinguishable from real concurrent plans, and therefore are not fully supported by traditional project management approaches. Also, the ability to re-use work done in support of the contingency plans is not addressed with these project management methods. Additionally, since decision-making is not an instantaneous event, it is not easily represented by these traditional project management tools (Roberts, 1994). This implies that the complete PERT chart may not be available when the project begins. Decision-making is, however, a process which is driven by a need to gather information to make the decision (March, 1991). Often, this process requires a significant amount of effort and time. Previous decision support system designs (Stohr and Konsynski, 1992) attempt to assist in this effort by allowing the decision-maker to model the problem, establish weights for alternatives, and eventually extract a decision from the system. The process of building the initial model is not addressed. Further, no attempt is made to manage this modeling process.

Current approaches to contingency planning simply support a "what-if" analysis which is performed before a project plan is executed (Kartam, Tzeng, and Teng, 1993). The purpose of this what-if analysis is to optimize a set of discrete events rather than to manage a dynamic, group-collaborative, decision-based process. What-if analyses simply involve making a discrete decision before the tasks are undertaken, and they assume a stable environment (i.e., no residual uncertainty after task commencement). Because there is a need to provide a more 
dynamic approach to project planning, this proposed research will involve the support of competitive contingency planning in a group-collaborative environment. Competitive contingency plans differ from other contingency plans in that multiple solutions are enacted in competition with each other. Usually contingency plans can be stated in a sentence like "if A then B else C". Competitive contingency planning allows both B and C to be executed until the truth of A can be established. A simple example of these differing views of contingency planning would be the preparation of a conference presentation. Suppose we wish to make a conference presentation about a new Web-based software product, but we do not know whether the conference organizers are offering Internet connections, equipment to display computer screens, or simply overhead projectors. We prefer to demonstrate our product online using the World Wide Web and project the interface on a screen for the conference participants to see, but the conference organizers do not know if they can provide this type of equipment. We have three options for presenting our new system. First, we can do "screen dumps" of our interfaces and simply display them with the overhead projector. Second, we can give a typical presentation with overheads, and follow that later with a demonstration on a computer at a small "break-out" session. Third, we can perform the demo to the assembled participants using a Web-connected computer and computer screen projection equipment. As the time for the conference approaches, we must proceed with preparations for our presentation, although the conference organizers still are unsure about the resources we will have available. Because we do not want to risk preparing only an elaborate on-line presentation that we may not be able to give, we decide to pursue all three competing contingencies. We prepare a Web-based presentation, and from this prepare overhead transparencies that we can use if a live demonstration is not feasible. It is possible that the time spent preparing transparencies will be wasted if we can do our Web-based demo, but we determine that risk to be acceptable. Regardless of our ultimate course of action, the time spent preparing the on-line demo is not likely to be wasted, because it is used in all three scenarios (it is used to generate screen dumps, it is used for the small demo in the break-out session, and it is used if the full-blown demo can be given). We call this type of task a "robust" task.

Contingency planning does provide a means for managing risk by allowing the enactment of a plan based upon the best available information at any particular time. However, as shown in the example, some wasted effort could result. So, some competing plans require more resources before they can be enacted, or alternately it may be necessary to use fewer resources per plan (e.g., use fewer people to prepare the full demo). Good management includes knowing how to control this risk and knowing which contingencies are appropriately undertaken. Selection of contingencies is a complex problem which relates to schedules and deadlines, utilization of resources (e.g., an employee is hired before enough is known about the project for correct tasking, so the employee starts work on a contingency), changing requirements, reliability of available information, impact upon or by external organizations, lack of domain knowledge, maximization of learning experiences, and minimization of cost by reuse of the work performed. Therefore, contingency planning can be seen to be both a valid response to project uncertainty, and affected by these aspects of project uncertainty as well.

To support decision management scenarios in which competing contingency plans are enacted, we propose a paradigm which must allow multiple decisions to be enacted simultaneously, and must allow the evaluation of multiple alternatives against a set of criteria. 
Further, the paradigm must support analysis of the tasks involved in each alternative which is enacted. Contingency plans are the result of a lack of information; therefore, new information from the tasks and the environment must be propagated to the team and to the manager in order to allow de-activation of contingency plans which are no longer relevant. This analysis of tasks should also support risk assessment based upon the "robustness" of contingent tasks. A "robust" task within a contingency plan, as illustrated previously, is one which is present in all contingencies; that is, it is performed regardless of which contingencies are enacted. Enactment of non-robust tasks carries a certain degree of risk which can be characterized by the probability that the contingency plan of which this task is a part is discarded. The potential of wasted effort which is put into a non-robust plan is itself one of the criteria that must be considered in choosing a contingency. Effort spent on a feasible contingency, which is later determined to be non-optimal, will often dictate the outcome. If a task appears in many contingencies, the risk is less than if it appears in only one contingency plan. If a task occurring in a single plan is performed, and then the plan is later abandoned, the work performed for this task is lost. If a task appears in several plans, it is more likely that the work performed will not be wasted.

Since each task in a contingency plan carries some risk of wasted effort, we can assign a probability value to the task representing this risk. We refer to this as the probability of wasted effort, or $\pi_{w}{ }^{i}$. This probability value is computed from the number of contingency plans in which the task appears as follows:

$\pi_{w}{ }^{i}=1$ - (number of contingencies in which task $i$ appears / total number of contingencies).

For example, if four competing contingencies have been enacted, and a task appears in three of them, the value of $\pi_{w}{ }^{i}$ would equal 1-(3/4), or $25 \%$.

The analysis must also include evaluation of tasks shared among contingencies in terms of schedular and resource constraints. Adjustment of project schedules may be necessary to allow resources to work on these simultaneous tasks, and to exploit the availability of the necessary resources.

\subsection{The Justification Matrix}

The justification matrix provides structure to organize the various activities of the decision management process, and is particularly useful when managing decisions needed for competitive contingency planning. It also contains the primitives which are used in the decision-making process. These primitives include the alternatives (the columns of the matrix), and the decision criteria (the rows of the matrix). This justification data structure also contains fields which denote contingency plans (which can be stored in a project management software system or in another relation in the database). As the process of decision-making proceeds, rows and columns of the matrix are filled, and contingencies are tagged. Instantiating the matrix forces a comprehensive examination of the problem, promotes in-depth understanding of the relevant decision issues, has historical value, and captures a growing body of knowledge as a problem is evaluated. The contents of the matrix depend on the type of the decision being made. For example, budgetary decisions will often have "hard" (quantifiable) criteria such as cost savings or anticipated expenditures for each alternative. Personnel-related decisions will 
have softer criteria, such as improved morale or development of better work habits. Although we have not done so, it is likely that a basic set of criteria can be defined for each classification. These sets could be reused as justification matrices are developed for each new decision.

\subsection{Group Communication For Contingency Planning}

Project decision-makers require the most current information possible as they attempt to guide the project. Making each project-related decision requires the elimination of uncertainty by gathering information relevant to the decision. It also requires that the decision-makers in the group have the most current information possible about the status of each competitive contingency plan which has been enacted, and about trigger points which have been reached. The use of competitive contingency plans demands that team members are constantly aware of the internal and external project environment, so that decisions made by the group are informed and effective.

To support this group communication, clear channels of communication between the project manager and team members must be maintained, along with communication among the team members themselves. Commercial project management and group collaboration software developers have recognized the necessity of communication among team members, and support this with various e-mail enhancements. We also believe that group communication is a pervasive process, and define a process for effective group communication in the presence of contingency plans. This process focuses information flow around project decisions, so that uncertainty is reduced as efficiently as possible.

The communication model is based upon seven types of messages, which are directed to team members whose identifiers are resident in the decision database. The first message, a decision request (DR), is sent to the relevant decision-maker when a decision must be made that is outside the recognized domain of the requester. The decision-maker then responds to the requester with a Response to DR (RDR), which either contains a decision, advises that no decision is forthcoming, advises of a date when the decision can be expected, or gives a probability that a particular course of action will be taken. A user can also pose a Question (Q) to any team members, and a response is give by team members in an Information message (I). "I" messages can also be used to pass any information or data to others in the cyberorganization. Users can also make Status Queries (SQ) to find out the status of a Decision Request if the request has been pending too long. Another feature of the communication model is the ability of a user to assess consensus within the cyberorganization about a decision issue by issuing a Gauge Interest (GI). Others in the cyberorganization respond to this message with Response to GI (RGI) messages, and the original requester can evaluate the relevance of the issue to the cyberorganization as a whole.

The seven message types, DR, RDR, I, Q, SQ, GI, and RGI, are denoted in the mail header, and the messages are counted if necessary to determine demand for a particular decision. This communication model's implementation is currently being studied, and will most likely be implemented in the context of groupware or networked mail tools. 


\section{THE UNCERTAINTY REDUCTION PROTOCOL AND ITS IMPLEMENTATION}

The use of Competitive Contingency Planning and the group communication paradigm discussed previously have as their stated goals the reduction of uncertainty in the cyberorganization. We propose a process for using these tools, and refer to this application as the Uncertainty Reduction Protocol (URP). The Uncertainty Reduction Protocol attempts to minimize uncertainty in the execution of project tasks by focusing team members' attention on project decisions and the resulting tasks. It also supports enactment of tasks even when decisions cannot be made due to lack of information or lack of consensus. The process presented here has two components. The first addresses a process for considering new decisions, and the second addresses the revisiting of decisions which have been previously entered into the system.

\subsection{Process for Using the Protocol}

The process for contingency planning is based upon the process used for the Decision-based System Development (DBSD), but carries it further. The process steps for newly introduced problems can be listed as follows:

- Identify and articulate the problem to be solved. In the URP implementation, this is done through a decision form interface.

- Identify alternative solutions. These are entered in the justification matrix.

- Choose decision criteria. These are entered in the justification matrix.

- Justify alternatives, using the data in the justification matrix.

- Plan contingencies, using project management software.

- Enact contingency plans, utilizing techniques defined in communication model. To obtain information about the status of pending decisions, the requester sends a status query to the person from whom the decision is needed. To request a decision from elsewhere in the cyberorganization, a decision request is submitted to the person(s) who must make the decision. An information message is sent when project information must be disseminated to others in the cyberorganization. Gauge interest messages are sent if consensus-building is required. A question message is sent as information is gathered, especially early in the enactment. A response to decision request message is sent as decision requests are addressed. This message can contain a decision, the date a decision can be expected, advice that no decision will be made, or the probability that a particular course of action will be followed. A response to a gauge interest message can be sent to inform original requesters of interest in the plan being enacted.

During this step, data regarding risk are collected, and trigger points are tracked with the project management system. The contingencies are tracked in the project management system for as long as they exist.

- Evaluate contingency plans, using the project management system (cost, resource, schedular analyses) and using the decision database to track pending decisions' progress. Information about progress of the plans may come from elsewhere in the 
cyberorganization, and these data are entered into the decision database and the project management package as necessary.

- Evaluate conditional decisions, and enter the effectiveness of the decision or contingency into the decision database.

One simple example of the use of this process would be the conference presentation example given in section 2.1 Competing Contingency Plans. Suppose we wish to enter our conference presentation contingency plan into our system. First, we prepare a concise statement describing the need to prepare this presentation, and our uncertainty about available resources for it. We type this into our decision database using the available form. Next, we identify the three alternative solutions for this problem (using transparencies, an off-line demo, and a full demo) and type these into the justification matrix spreadsheet. The criteria we will be using to select our presentation medium are listed in the justification matrix (namely, the availability of an appropriate platform and the availability of a network connection). Then, we plan our contingencies by listing tasks for all three alternatives in our favorite project management software, and we link the tasks to the decision database (we use Time Line Solutions' TimeLine ${ }^{\circledR}$ product for project management, and we take advantage of the project management software's Object Linking and Embedding (OLE) capability to accomplish this). Then, we proceed to prepare the three presentations, after confirming that we have adequate resources to do this. Finally, after reaching the conference site, we can evaluate the contingencies by determining which hardware resources are available, and select one presentation from our contingencies. We then enter this selection into the decision database for future reference in case we submit a paper for this conference again at a later time.

Revisiting decisions is usually prompted by the receipt of information from elsewhere in the cyberorganization. It can result from the receipt of a structured message as required by the group communication model when contingencies are enacted. The communication model defines how this message is handled.

If incoming information is unstructured, or comes from a source other than the email system, the following process is used to handle the information:

- The decision database is queried for a list of all decisions, or an SQL query can be used to search for a known keyword. The user extracts pertinent decisions and reviews them. The information obtained is entered into the justification matrix and decision database as appropriate.

- A "closure generator" is then used to trace through other related decisions. This tool traces through the decision database to find all decisions which are logically linked (i.e., parent decisions which spawned sub-decisions, etc.). The new information is entered in any relevant decisions.

- The new information is then placed into the project management tool if it involves schedule, budget, or resources. The appropriate tasks can be found from links from the decision database.

- A "mail propagation tool" is next used if others in the cyberorganization must be notified of changes resulting from the new information. The contact list from the decision database is used to determine to whom the information message should be propagated. 
- If the new information requires that a decision should be made elsewhere in the cyberorganization, the decision request is generated and sent.

- If the new information enables response to a decision request, the response to decision request is sent.

- If the new information raises questions, the question message is prepared and sent using the mail propagation tool.

- If the new information spawns contingencies, they are entered as described in the previous section. The probability of wasted effort is calculated for the plans by the system, and this information can be used to analyze the contingencies' risks. The process then follows the steps outlined for new problems.

As an example, referring again to the conference presentation example, suppose we are preparing the three presentation formats as outlined. Before we have completed the preparation of the demonstrations, we receive a letter from the conference organizers informing us that we will only have an overhead projector available to us for our presentation at the conference site. Therefore, we can use this information to select the single contingency which is appropriate: the preparation of a transparencies. We search the decision database for the relevant problem using the query mechanism we have available, then we enter the decision which was made (i.e., to use transparencies for our presentation). We update the tasks in the project management tool, then use the mail propagation software to inform the team members to concentrate on the development of the presentation using the overhead projector only.

\subsection{Implementation of the Protocol}

The implementation of the Uncertainty Reduction Protocol is being done in the context of enhancements to commercially available software products. The main reason for this approach is so users will feel comfortable using the protocol in the familiar context of project management software, spreadsheet software, and groupware. The architecture being implemented consists of a decision database, which can be maintained in any ODBC-compliant database or spreadsheet system, a communication form implemented in groupware, a decision interface, the justification matrix (currently implemented in a Microsoft Excel $\otimes^{\circ}$ spreadsheet), and data from the user's project management software package (we are using Time Line Solutions Corporation's TimeLine ${ }^{\circledR}$ project management software). The user is expected to use any problem-solving methodology he or she wishes. The architecture is illustrated in Figure 1 .

By using the protocol as described in the process outlined previously, and an implementation of the proposed architecture, we would expect the system to answer user questions such as the following:

1. I receive an email relating to a decision. It has a predictable structure containing keywords. How do I find decisions relevant to this email in the decision database?

2. I receive an email or phone call relating to a decision, or I got information about the decision in a meeting. The information is not structured and may not be in the system at all. How do I find decisions which are relevant to this unstructured message? 
3. I make or change a decision. Which other decisions and tasks are affected by this modification?

4. I am tracking competing contingencies with a project management tool. New information arrives about these contingency plans. What happens?

5. I am tracking competing contingencies with a project management tool. A trigger point is encountered. What happens?

6. I make or change a decision and need to alert others in the cyberorganization with needto-know. How is this accomplished?

7. I need some consensus in the cyberorganization about a pending decision I must make. How do I get this?

8. I made an important decision which should be made known to some other people in the cyberorganization. How do I do this? How do I find out which people have need-toknow?

9. I have to make a decision, and I wonder who else in the cyberorganization cares about it. How do I find out?

10. I need my management to make a decision for me. How do I request this?

11. I need to know the status of a decision I requested. How do I find out?

12. I got a Gauge Interest (GI) message about a decision I'm interested in working on. How do I let the sender know that I'm out here and interested?

13. I need some information from elsewhere in my cyberorganization before I can make a decision. How to I get it?

14. I need to change my schedule and resource allocation for the project. Which decisions are affected?

15. I need to change some decisions I made. How is the schedule/resource allocation/budget changed to correctly reflect this? How do I pick up all decisions in this "closure"?

16. How do I know how robust a task is (i.e., what is the value of $\pi_{w}{ }^{i}$ )?

17. I need a list of all decisions that have to do with " $x$ ".

18. I need to know the financial and schedular risk of pursuing a couple of contingency plans. How do I get this?

19. How do I optimize contingency plans so that I waste the least work?

20. What is the global uncertainty of my project?

21. I can't make up my mind about " $\mathrm{z}$ ". What is this indecision costing me? Do I have to make a decision, or can I afford to defer it?

Answering user questions such as these requires the presence of some new system features. These features include a mail handler, which builds a query upon receipt of a structured message, and then searches the database for decisions based on the keywords in the message. A decision list generator is needed for presenting the user with a comprehensive list of decisions if a structured query cannot be build from the received message. A closure generator should trace up and down links in the decision database and present the user with a list of decisions which are related logically in the database. A mail propagation tool uses the contact list in the database to send structured messages to relevant people in the cyberorganization. Finally, the computation of the wasted effort in a contingency plan should be performed for the user upon demand. We are currently implementing these features and will subsequently evaluate their usefulness. 
Groupware

Envelope

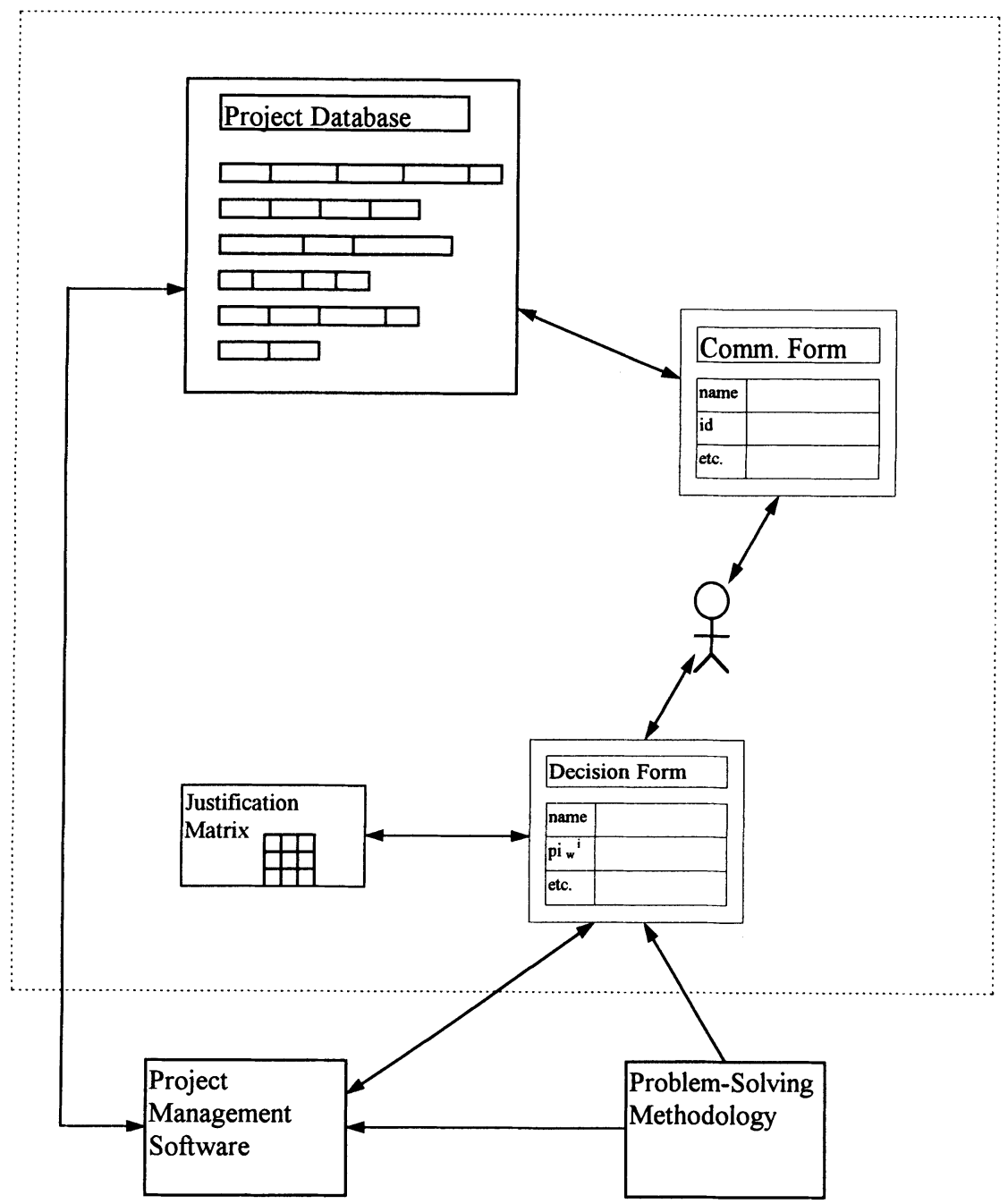

Figure 1 Uncertainty Reduction Protocol Architecture 


\section{SUMMARY}

This paper has discussed processes for contingency planning and group communication which support the management of uncertainty in large system development and engineering projects.

We believe that the responding to project uncertainty with enactment of competitive contingency plans is a widely-used project management technique which is not currently wellsupported. We propose a decision-centered process with a supporting information and communication structure for managing contingency planning. This ensures that team members in a decision-making capacity are aware of all current information about each of their impending decisions, and that they know when contingency plans have been resolved.

Future work will involve the evaluation of the success of this application. We anticipate revisions of the Uncertainty Reduction Protocol as necessary based upon these evaluation studies.

\section{REFERENCES}

Kartam, N., Tzeng, G.-H., and Teng, J.-Y. (1993) Robust Contingency Plans for Transportation Investment Planning. IEEE Transactions on Systems, Man, and Cybernetics, 23:1, 5-13.

Kraut, R., and Streeter, L. (1995) Coordination in Software Development. Communications of the $A C M, 38: 3,69-81$.

March, J. (1991) How Decision Happen in Organizations. Human-Computer Interaction, 6, 95-117.

Roberts, C. (1994) A Decision-based System Development Paradigm for Support of Contingency Planning and Group Collaboration. Old Dominion University Ph.D. Thesis Proposal.

Stohr, E., and Konsynski, B. (1992) Information Systems and Decision Processes. IEEE Computer Society Press, Los Alamitos, CA.

Wild, C., and Maly, K. (1992) Software Life Cycle Support - Decision-based Software Development. Information Processing 92, 1, 72-78.

Wild, C., Maly, K., Zhang, C., Roberts, C., Rosca, D., and Taylor, T. (1994) Project Management Using Hypermedia CASE Tools. 1994 Conference on Data and Knowledge Systems for Manufacturing and Engineering, 722-727.

Winstanley, G. (1991) Artificial Intelligence in Engineering. John Wiley and Sons, New York.

\section{BIOGRAPHY}

Cathy Roberts is a Ph.D. candidate in Computer Science at Old Dominion University. She received her B.S. degree in Industrial Engineering and Operations Research from Virginia Tech in 1983, and her M.S. degree in Computer Science from Old Dominion University in 1992. She has held industrial engineering, systems engineering, and project management positions in 
industry. Her current research interests include decision-based systems development, project management issues, and software engineering.

Chris Wild received his Ph.D. degree in Computer Science from Rutgers University in 1977 in the area of computer vision. He worked for eight years at Bell Telephone Laboratories on computer graphics, robotics, $\mathrm{CAD} / \mathrm{CAM}$, data base and communications network systems. He is currently an associate professor at Old Dominion University. His research interests are software testing, decision-based systems development, distance learning and medical support systems.

Kurt J. Maly received the Dipl. Ing. degree from the Technical University of Vienna, Austria, and the M.S. and Ph.D. Degrees from the Courant Institute of Mathematical Sciences, New York University, New York, NY. He is Kaufman Professor and Chair of Computer Science at Old Dominion University, Norfolk, VA. Before that, he was at the University of Minnesota, both as faculty member and Chair. He also is Visiting Professor at Chengdu University of Science and Technology, People's Republic of China and is Honorary Professor at Hefei University of Technology, PRC. His research interests include modeling and simulation, very high-performance networks protocols, reliability, interactive multimedia remote instruction, Internet resource access, and software maintenance. 